\title{
A NOTE ON THE ESSENTIAL SELFADJOINTNESS OF CLASSICAL CONSTANTS OF MOTION
}

\author{
BENT ØRSTED
}

\begin{abstract}
It is shown how the results by Chernoff [1] and also Rauch and Taylor [2] on the essential selfadjointness of powers of generators of hyperbolic mixed problems can be combined with results by Poulsen [3] to give essential selfadjointness of symmetric operators commuting with the hyperbolic problem, as specified below.
\end{abstract}

Let $M$ be a complete Riemannian manifold with volume element $d v$ and $\xi$ a hermitian bundle over $M$ with $\langle\cdot, \cdot\rangle_{x}$ the inner product in the fiber over $x$. As in [1] we consider a first-order symmetric hyperbolic operator $L$ on $C^{\infty}(\xi)$, all $C^{\infty}$ sections of $\xi$, of the form

$$
L=\sum_{i=1}^{n} A^{i}(x) \frac{\partial}{\partial x_{i}}+B(x) .
$$

Under the assumption that the local velocity of propagation of solutions to $\partial u / \partial t=L u$ is sufficiently low, we get [1] that this has unique global solutions given initial data in $C_{0}^{\infty}(\xi)$, all $C^{\infty}$ sections with compact support. Hence we infer the existence of a continuous unitary one-parameter group $V(t)$ in $H=L^{2}(\xi)$ such that

$$
\begin{aligned}
(d / d t) V(t) u & =V L(t) u=V(t) L u, \\
V(t) u & \in C_{0}^{\infty}(\xi)
\end{aligned}
$$

for all $u \in C_{0}^{\infty}(\xi)$. In particular, the generator of $V(t)$ contains $i L$.

LEMMA 1. Let $U$ be a continuous unitary representation of a Lie group $G$ in a Hilbert space $H$ and $D_{\infty}$ the space of $C^{\infty}$ vectors for $U$ [3]; assume that $D$ is a group-invariant dense subspace of $H$ contained in $D_{\infty}$. Suppose that $T$ is a symmetric operator defined on $D_{\infty}$ such that $T U(g) \supseteq U(g) T$ for all $g \in G$. Then $T$ is essentially selfadjoint on $D$.

Proof. From [3, Theorem 1.3] it follows immediately that $D$ is dense in $D_{\infty}$ with its natural Fréchet topology, and from [3, Corollary 2.2] that $T$ is

Received by the editors April 12, 1976.

AMS (MOS) subject classifications (1970). Primary 35L40, 47B25; Secondary 58G99, 22 E45.

Key words and phrases. Hyperbolic generators, essential selfadjointness of constants of motion, unitary representations of Lie groups.

Copyright $\odot$ 1977, American Mathematical Suciety 
essentially selfadjoint. Let $\left(D_{\infty}, \tau\right)$ denote $D_{\infty}$ equipped with its natural Fréchet topology and consider $T$ as an operator $S$ from $\left(D_{\infty}, \tau\right)$ to $H$; since $T$ is symmetric it is closable and it follows easily that $S$ is closed. By the closed graph theorem $S$ is actually continuous, so for each $y \in D_{\infty}$ there is a sequence $\left\{x_{n}\right\}_{n \in N} \subseteq D$ such that $\left\{x_{n}\right\}$ converges to $y$ in $D_{\infty}$ and $\left\{T x_{n}\right\}$ converges to $T y$ in $H$. But this means that $T \uparrow D_{\infty} \subseteq(T \uparrow D)^{-}$, and, hence, $(T \uparrow D)^{-}=\left(T \uparrow D_{\infty}\right)^{-}$is selfadjoint. Q.E.D.

COROLlaRY 2. Let $T$ be a symmetric operator in $H=L^{2}(\xi)$ defined on the $C^{\infty}$ vectors for the group $V(t)$ above. Suppose that $T V(t) \supseteq V(t) T(T \in \mathbf{R})$. Then $T$ is essentially selfadjoint on $C_{0}^{\infty}(\xi)$.

Proof. As the domain $D$ we take $C_{0}^{\infty}(\xi)$ and directly apply the lemma. Q.E.D.

If we interpret $i L$ as the hamiltonian of a quantum mechanical system with Hilbert state space $H$, we see that the result asserts the essential selfadjointness of those constants of motion which are defined on the $C^{\infty}$ vectors for $V(t)$.

The lemma above may also be applied to the case of a second order wave equation to yield essential selfadjointness of operators commuting with $-\Delta+g(x)$ on $\mathbf{R}^{n}$. Specifically we have

CoRollary 3. Let $g$ be $C^{\infty}$ and $T=-\Delta+g(x)$ semibounded on $L^{2}\left(\mathbf{R}^{n}\right)$, and let $D_{\infty}$ be the largest subspace where all powers of $T$ are defined. Suppose $T_{1}$ is symmetric and defined on $D_{\infty}$ such that $T_{1} T=T T_{1}$ on $D_{\infty}$. Then $T_{1}$ is essentially selfadjoint on $C_{0}^{\infty}\left(\mathbf{R}^{n}\right)$.

\section{REFERENCES}

1. P. Chernoff, Essential self-adjointness of powers of generators of hyperbolic equations, J. Functional Analysis 12 (1973), 401-414. MR 51 \#6119.

2. J. Rauch and M. Taylor, Essential self-adjointness of powers of generators of hyperbolic mixed problems, J. Functional Analysis 12 (1973), 491-493. MR 50 \#82.

3. N. S. Poulsen, On $C^{\infty}$-vectors and intertwining bilinear forms for representations of Lie groups, J. Functional Analysis 9 (1972), 87-120. MR 46 \# 9239.

Department of Mathematics, Massachusetts institute of Technology, Cambridge, MASSACHUSETTS 02139 